\title{
Effects of Thermophoresis, Viscous Dissipation and Joule Heating on Steady MHD Flow over an Inclined Radiative Isothermal Permeable Surface with Variable Thermal Conductivity
}

\author{
M. G. Reddy \\ Department of Mathematics, Acharya Nagarjuna University Campus, Ongole, A.P- 523 001, India \\ Email:mgrmaths@gmail.com
}

(Received April 27, 2012; accepted February 6, 2013)

\begin{abstract}
A two-dimensional mathematical model is presented for the laminar heat and mass transfer of an electricallyconducting, viscous and Joule (Ohmic) heating fluid over an inclined radiate isothermal permeable surface in the presence of the variable thermal conductivity, thermophoresis and heat generation. The Talbot- Cheng-SchefferWillis formulation (1980) is used to introduce a thermophoretic coefficient into the concentration boundary layer equation. The governing partial differential equations are non-dimensionalized and transformed into a system of nonlinear ordinary differential similarity equations, in a single independent variable $\eta$. The resulting coupled nonlinear equations are solved under appropriate transformed boundary conditions using the Runge-Kutta fourth order along with shooting method. Comparisons with previously published work are performed and the results are found to be in very good agreement. Computations are performed for a wide range of the governing flow parameters, viz., magnetic field parameter, thermophoretic coefficient (a function of Knudsen number), Eckert number (viscous heating effect), angle of inclination, thermal conductivity parameter, heat generation parameter and Schmidt number. The present problem finds applications in optical fiber fabrication, aerosol filter precipitators, particle deposition on hydronautical blades, semiconductor wafer design, thermo-electronics and magnetohydrodynamic energy generators.
\end{abstract}

Keywords: Thermophoresis, Magnetohydrodynamics, Heat and mass transfer, Thermal radiation, Viscous dissipation, Joule heating, Heat generation, Inclined plate

\section{NOMENCLATURE}

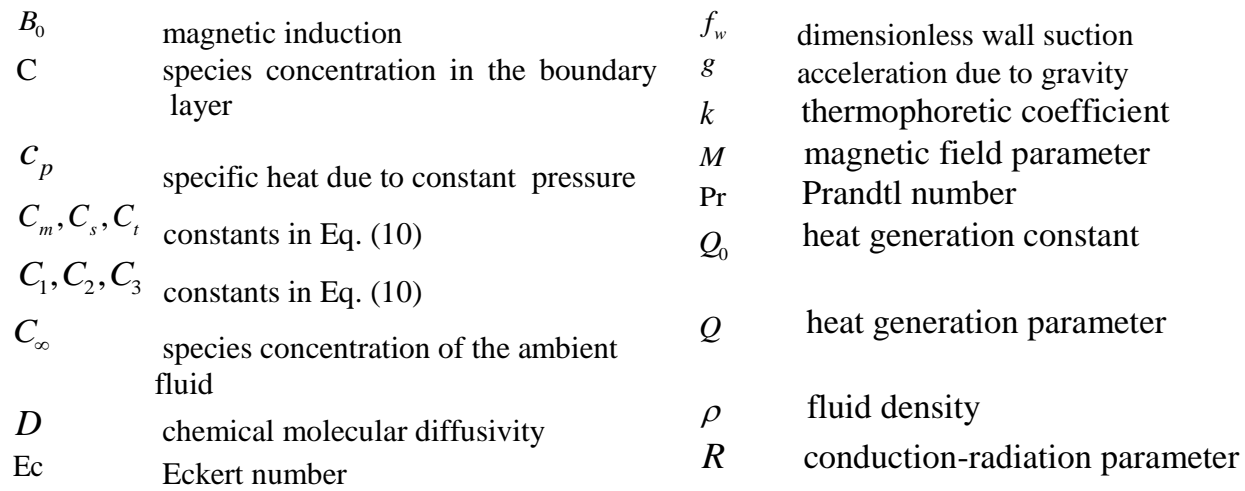




\begin{tabular}{|c|c|}
\hline $\mathrm{Re}_{x}$ & local Reynolds number \\
\hline Sc & Schmidt number \\
\hline$T$ & $\begin{array}{l}\text { temperature of the fluid in the } \\
\text { boundary layer }\end{array}$ \\
\hline$T_{\infty}$ & temperature of the ambient fluid \\
\hline$T_{w}$ & temperature at the surface \\
\hline$U_{\infty}$ & free stream velocity \\
\hline$u, v$ & $\begin{array}{l}\text { velocity components in } x, y \\
\text { directions }\end{array}$ \\
\hline$v_{m}(x)$ & transpiration velocity \\
\hline$V_{T}$ & thermophoretic velocity \\
\hline$x, y$ & $\begin{array}{l}\text { axis in direction along and normal to } \\
\text { the plate }\end{array}$ \\
\hline$\alpha$ & angle of inclination to vertical \\
\hline$\beta$ & $\begin{array}{l}\text { volumetric coefficient of thermal } \\
\text { expansion }\end{array}$ \\
\hline
\end{tabular}

\section{INTRODUCTION}

Thermophoresis is the migration of aerosol and other particles in the direction of a decreasing temperature gradient. Such a phenomenon has received considerable attention in the engineering analysis community owing to major applications in optical fiber production, heat exchanger fouling, aerosol reactors etc. In optical fiber synthesis, thermophoresis has been identified as the principal mechanism of mass transfer as used in the technique of modified chemical vapour deposition (MCVD) Kremer et al. (2003). In this procedure a gaseous mixture of reactive precursors is directed over a heated substrate where solid film deposits are located. In particular the mathematical modeling of the deposition of silicon thin films using MCVD methods has been accelerated by the quality control measures enforced by the micro-electronics industry. Such topics involve a variety of complex fluid dynamical processes including thermophoretic transport of particlauet deposits, heterogenous/homogenous chemical reactions, homogenous particulate nucleation and coupled heat and energy transfer. Boundary layer theory has proven to be instrumental in simplifying the flow regimes to facilitate numerical solutions via CFD and also userspecified numerical codes. Thermophoresis is also a key mechanism of study in semi-conductor technology, especially controlled high-quality wafer production as well as in radioactive particle deposition in nuclear reactor safety simulations and MHD energy generation system operations. A number of analytical and experimental papers in thermophoretic heat and mass transfer have been communicated. Brock (1962) provided an early analysis of aerosol thermophoretic dynamics. Batchleor and Shen (1985) later analyzed the thermophoretic migration of particles in a gaseous flow. Goren (1977) considered the thermophoretic deposition of particles in flat plate boundary layers. Talbot et al. (1980) presented a seminal study, considering boundary layer flow with thermophoretic effects, which has become a benchmark for subsequent studies (this model is extended in the present paper). The thermophoretic flow of larger diameter particles was investigated by $\gamma \quad$ local buoyancy parameter

$\sigma \quad$ electrical conductivity

$\sigma_{1}$ Stefan-Boltzmann constant stream function similarity variable

kinematic viscosity

$\mu \quad$ fluid viscosity

$\tau_{w} \quad$ wall shear stress

$\theta$ dimensionless temperature

$\phi \quad$ dimensionless concentration

$\lambda_{g}$ thermal conductivity of fluid

$\lambda_{p} \quad$ thermal conductivity of diffused

$\tau \quad$ thermophoretic parameter

$\chi \quad$ mean absorption coefficient

( ) differentiation with respect to $\eta$

Kanki et al. (1985). Lin and Ahn (1987) studied thermophoretic flows in semi-conductor materials. Shen (1988) discussed thermophoresis in two-dimensional and axisymmetric flow near cooled bodies. Sasse et al. (1992) considered laminar thermophoretic flows in various flat surface and concentric geometries.

The study of magnetohydrodynamic viscous radiate flows has important industrial, technological and geothermal applications such as high-temperature plasmas, cooling of nuclear reactors, liquid metal fluids, MHD accelerators, and power generation systems. Hossain and Takhar (1996) analyzed the effect of radiation using the Rosseland diffusion approximation which leads to non-similar solutions for the forced and free convection flow of an optically dense fluid from vertical surfaces with constant free stream velocity and surface temperature. Hossain et al. (1999) studied the effect of radiation on free convection heat transfer from a porous vertical plate. Duwairi and Damseh (2004) studied the radiation-conduction interaction in free and mixed convection fluid flow for a vertical flat plate in the presence of a magnetic field effect. Mbeledogu and Ogulu (2007) studied the heat and mass transfer of an unsteady MHD natural convection flow of a rotating fluid past a vertical porous flat plate in the presence of radiative heat transfer. Rahman and Sattar (2007) studied the transient convective heat transfer flow of micropolar fluid past a vertical porous plate in the presence of thermal radiation. Alam et al. (2008) studied the effects of variable suction and thermophoresis on steady MHD combined free-forced convective heat and mass transfer flow over a semiinfinite permeable inclined plate in the presence of thermal radiation. Gnaneswara Reddy and Bhaskar Reddy (2010) studied the radiation and mass transfer effects on unsteady MHD free convection flow past a vertical porous plate with viscous dissipation by using finite element method. Recently, Gnaneswara Reddy and Bhaskar Reddy (2011) investigated mass transfer and heat generation effects on MHD free convection flow past an inclined vertical surface in a porous medium. Lie group analysis of heat and mass transfer 
effects on steady MHD free convection flow past an inclined surface with viscous dissipation investigated by Gnaneswara Reddy (2013). Gnaneswara Reddy (2012) analyzed lie group analysis of heat and mass transfer effects on steady MHD free convection dissipative fluid flow past an inclined porous surface with heat generation.

The viscous and joule heating of ionized gases on forced convection heat transfer in the presence of magneto and thermal radiation effect was investigated by Duwairi (2005). Recently, Osalusi et al. (2007) studied the effectiveness of viscous dissipation and Joule heating on steady MHD flow and heat transfer of a Bingham fluid over a porous rotating disk in the presence of Hall and ion-slip currents.

The objective of the present paper is to study the combined effects of viscous dissipation and Joule heating on steady magnetohydrodynamic free convective heat and mass transfer flow of a viscous incompressible fluid past a semi-infinite inclined radiate isothermal permeable moving surface in the presence of thermophoresis and heat generation with variable thermal conductivity.

\section{GOVERNING EOUATIONS}

Consider a two-dimensional steady magnetohydrodynamic laminar free convective heat and mass transfer flow of a viscous incompressible and electrically conducting fluid past a continuously moving semi-infinite inclined porous flat plate with an acute angle a to the vertical with surface temperature $T_{w}$, surface concentration $C_{w}$, both constant and thermal conductivity, $k_{f}$, which obeys a linear temperature law according to $k_{f}=k_{0}\left[1+\alpha\left(T-T_{\infty}\right)\right]=k_{0}(1+\beta \theta) \quad$ where $\quad k_{0}$ denotes thermal conductivity in the free stream of the flow, $\alpha$ is a thermophysical constant dependent on the fluid ( $\alpha<0$ for lubrication oils, hydromagnetic working fluids and $\alpha>0$ for air or water) and $\beta=\alpha\left(T_{w}-T_{\infty}\right)$ is the thermal conductivity variation parameter. The $x$-axis measured along the plate, a magnetic field of uniform strength $B_{0}$ is applied in the $y$-direction that is normal to the flow direction. Fluid suction or injection is imposed at the plate surface. The temperature of the surface is held uniform at $T_{w}$ which is higher than the ambient temperature $T_{\infty}$. The species concentration at the surface is maintained uniform at $C_{w}$, which is taken to be zero and that of the ambient fluid is assumed to be $C_{\infty}$. The effect of thermophoresis and variable thermal conductivity is being taken into account to help in the understanding of the mass deposition variation on the surface. We further assume that (i) the mass flux of particles is sufficiently small so that the main stream velocity and temperature fields are not affected by the thermophysical processes experienced by the relatively small number of particles, (ii) due to the boundary layer behavior the temperature gradient in the $y$-direction is much larger than that in the $x$-direction and hence only the thermophoretic velocity component which is normal to the surface is of importance, (iii) the fluid has constant kinematic viscosity and thermal diffusivity, and that the Boussinesq approximation may be adopted for steady laminar flow, (iv) the particle diffusivity is assumed to be constant, and the concentration of particles is sufficiently dilute to assume that particle coagulation in the boundary layer is negligible, (v) the magnetic Reynolds number is assumed to be small so that the induced magnetic field is negligible in comparison to the applied magnetic field and (vi) the fluid is considered to be gray; absorbing-emitting radiation but non-scattering medium and the Rosseland approximation is used to describe the radioactive heat flux in the $x$ direction is considered negligible in comparison to the $y$-direction.

Under the above assumptions, the governing equations for this problem can be written as

Continuity equation

$\frac{\partial u}{\partial x}+\frac{\partial v}{\partial y}=0$

Momentum equation

$u \frac{\partial u}{\partial x}+v \frac{\partial u}{\partial y}=v \frac{\partial^{2} u}{\partial y^{2}}+g \beta\left(T-T_{\infty}\right) \cos \alpha-\frac{\sigma B_{0}^{2}}{\rho} u$

Energy equation

$$
\begin{aligned}
& u \frac{\partial T}{\partial x}+v \frac{\partial T}{\partial y}=\frac{1}{\rho c_{p}} \frac{\partial}{\partial y}\left(k_{f} \frac{\partial T}{\partial y}\right) \frac{\partial^{2} T}{\partial y^{2}} \\
& +\frac{\mu}{\rho c_{p}}\left(\frac{\partial u}{\partial y}\right)^{2}-\frac{1}{\rho c_{p}} \frac{\partial q_{r}}{\partial y}+\frac{\sigma B_{0}^{2}}{\rho c_{p}} u^{2} \\
& +\frac{Q_{0}}{\rho c_{p}}\left(T-T_{\infty}\right)
\end{aligned}
$$

Species equation

$u \frac{\partial C}{\partial x}+v \frac{\partial C}{\partial y}=D \frac{\partial^{2} C}{\partial y^{2}}-\frac{\partial}{\partial y}\left(V_{T} C\right)$

where $u$ and $v$ are the velocity components in the $x$, $y$ directions, respectively, $v$ is the kinematic viscosity, $\mu$ is the fluid viscosity, $g$ is the acceleration due to gravity, $\rho$ is the density of the fluid, $\beta$ is the volumetric coefficient of thermal expansion, $T$, $T_{w}$ and $T_{\infty}$ are the temperature of the fluid inside the thermal boundary layer, the plate temperature and the fluid temperature in the free stream, respectively, while $C, C_{w}$ and $C_{\infty}$ are the corresponding concentrations, $B_{0}$ is the magnetic induction, $\sigma$ is the electrical conductivity, $k_{f}$ is the thermal conductivity of fluid, $c_{p}$ is the specific heat at constant pressure, $q_{r}$ is the radiative heat flux in the $y$-direction, $Q_{0}$ is heat generation constant, $D$ is the molecular diffusivity of the species concentration and $V_{T}$ is the thermophoretic velocity. 
The appropriate boundary conditions for the above model are as follows:

$u=U_{0}, v= \pm v_{w} T=T_{w}, C=C_{w}$ at $y=0$

$u \rightarrow 0, T \rightarrow T_{\infty}, C \rightarrow C_{\infty}$ as $y \rightarrow \infty$

where $U_{0}$ is the uniform plate velocity and $v_{w}(x)$ represents the permeability of the porous surface. Here, we confine our attention to the suction/injection of fluid through the porous surface and for these we also consider that the transpiration function variable $v_{w}(x)$ is of the order of $x^{-1 / 2}$.

The radiative heat flux $q_{r}$ under Rosseland approximation has the form

$q_{r}=-\frac{4 \sigma_{s}}{3 k_{e}} \frac{\partial T^{4}}{\partial y}$

where $\sigma_{s}$ is the Stefan-Boltzmann constant and $k_{e}$ the mean absorption coefficient. It should be noted that by using the Rosseland approximation, the present analysis is limited to optically thick fluids. If the temperature differences within the flow are sufficiently small, then Eq. (6) can be linearized by expanding $T^{4}$ into the Taylor series about $T_{\infty}$, which after neglecting higher order terms takes the form

$T^{4} \cong 4 T_{\infty}^{3} T-3 T_{\infty}{ }^{4}$

Using Eqs. (6) and (7) in Eq. (3) we have

$$
\begin{aligned}
& u \frac{\partial T}{\partial x}+v \frac{\partial T}{\partial y}=\frac{1}{\rho c_{p}} \frac{\partial}{\partial y}\left(k_{f} \frac{\partial T}{\partial y}\right) \frac{\partial^{2} T}{\partial y^{2}} \\
& +\frac{\mu}{\rho c_{p}}\left(\frac{\partial u}{\partial y}\right)^{2}-\frac{16 \sigma^{*} T_{\infty}^{3}}{3 \rho c_{p} k_{e}} \frac{\partial^{2} T}{\partial y^{2}}+\frac{\sigma B_{0}^{2}}{\rho c_{p}} u^{2} \\
& +\frac{Q_{0}}{\rho c_{p}}\left(T-T_{\infty}\right)
\end{aligned}
$$

The second, third, fourth and fifth terms on the RHS of Eq. (8) denote the viscous, thermal radiation, magnetic heating and heat generation terms, respectively.

Now the thermophoretic velocity $V_{T}$, which appears in Eq. (4), can be written as (see Talbot et al. (1980)):

$V_{T}=-k v \frac{\nabla T}{T_{r}}=-\frac{k v}{T_{r}} \frac{\partial T}{\partial y}$

where $T_{r}$ is some reference temperature and $k$ is the thermophoretic coefficient which ranges in value from 0.2 to 1.2 as indicated by Batchelor and Shen (1985) and is defined from the theory of Talbot et al. (1980) by

$$
k=\frac{2 C_{s}\left(k_{f} / \lambda_{p}+C_{t} K n\right)\left[1+K n\left(C_{1}+C_{2} e^{-C_{3} / K n}\right]\right.}{\left(1+3 C_{m} K n\right)\left(1+2^{k_{f}} / \lambda_{p}+2 C_{t} K n\right)}
$$

where $C_{1}, C_{2}, C_{3}, C_{m}, C_{s}, C_{t}$ are constants, $k_{f}$ and $\lambda_{p}$ are the thermal conductivities of the fluid and diffused particles, respectively, and $K n$ is the Knudsen number.

A thermophoretic parameter $\tau$ can be defined (see Mills et al.; 1984 and Tsai; 1999) as follows:

$\tau=-\frac{k\left(T_{w}-T_{\infty}\right)}{T_{r}}$

Typical values of $\tau$ are $0.01, \quad 0.05$ and 0.1 corresponding to approximate values of $-k\left(T_{w}-T_{\infty}\right)$ equal to 3,15 and $30 K$ for a reference temperature of $T_{r}=300 \mathrm{~K}$.

The Eqs. (1), (2), (8) and (4) are strongly coupled, parabolic and nonlinear partial differential equations. An analytical solution cannot be obtained and therefore we seek numerical solutions. Numerical computations are greatly facilitated by non-dimensionalization of the equations. Proceeding with the analysis, we introduce the following similarity transformations and dimensionless variables which will convert the partial differential equations from two independent variables $(x, y)$ to a system of coupled, non-linear ordinary differential equations in a single variable $(\eta)$ i.e. coordinate normal to the plate. In order to write the governing equations and the boundary conditions in dimensionless form, the following non-dimensional quantities are introduced (Chamkha and Issa; 2000).

$$
\begin{gathered}
\eta=y \sqrt{\frac{U_{0}}{2 v x}}, \quad \psi=\sqrt{v x U_{0}} f(\eta), \\
\theta(\eta)=\frac{T-T_{\infty}}{T_{w}-T_{\infty}}, \phi(\eta)=\frac{C}{C_{\infty}}
\end{gathered}
$$

where $f(\eta)$ is the dimensionless stream function and $\psi$ is the dimensional stream function defined by

$u=\frac{\partial \psi}{\partial y}$ and $v=-\frac{\partial \psi}{\partial x}$

Then, introducing the Eq. (12) into Eq. (1), we obtain

$$
u=U_{0} f^{\prime}(\eta) \text { and } v=\sqrt{\frac{U_{0} v}{2 x}}\left(\eta f^{\prime}-f\right)
$$

Here, prime denotes ordinary differentiation with respect to the similarity variable $\eta$. Substituting Eqs. (12) and (14) in Eqs. (2), (8) and (4), we obtain the following non-linear differential equations

$$
\begin{aligned}
& f^{\prime \prime \prime}+f f^{\prime \prime}+\gamma \theta \cos \alpha-M f^{\prime}=0 \\
& \theta^{\prime \prime}(1+\beta \theta)+\beta \theta^{\prime 2}+\left(\frac{3 R \operatorname{Pr}}{3 R+4}\right)\left(f \theta^{\prime}\right. \\
& \left.+E c\left(f^{\prime \prime}\right)^{2}+E c M\left(f^{\prime}\right)^{2}+Q \theta\right)=0
\end{aligned}
$$


$\phi^{\prime \prime}+S c\left(f-\tau \theta^{\prime}\right) \phi^{\prime}-S c \tau \phi \theta^{\prime \prime}=0$

The non-dimensional parameters that appeared in the above equations are defined as follows:

$$
\begin{aligned}
& \gamma=\frac{G r_{x}}{\operatorname{Re}_{x}^{2}}, G r_{x}=\frac{g \beta\left(T_{w}-T_{\infty}\right)(2 x)^{3}}{v^{2}}, \operatorname{Pr}=\frac{v \rho c_{p}}{k_{f}}, \\
& M=\frac{2 \sigma B_{0}^{2} x}{\rho U_{0}}, \operatorname{Re}_{x}=\frac{U_{0} 2 x}{v}, E c=\frac{U_{0}^{2}}{c_{p}\left(T_{w}-T_{\infty}\right)}, \\
& R=\frac{k_{f} k_{e}}{4 \sigma_{s} T_{\infty}^{3}}, Q=\frac{2 Q_{0}}{\rho c_{p} U_{o}}, S c \frac{v}{D}
\end{aligned}
$$

The corresponding boundary conditions are

$$
\begin{aligned}
& f=f_{w}, f^{\prime}=1, \theta=1, \phi=0 \text { at } \eta=0 \\
& f^{\prime} \rightarrow 0, \theta \rightarrow 0, \phi \rightarrow 1 \quad \text { as } \quad \eta \rightarrow \infty
\end{aligned}
$$

where $f_{w}=-v_{w}(x) \sqrt{\frac{2 x}{v U_{0}}}$ is the permeability of the porous surface which is positive for suction and negative for injection. The parameters $\gamma, M, \operatorname{Pr}, E c$, $R, Q, \tau, S c$ are the local buoyancy parameter, magnetic field parameter, Prandtl number, Eckert number, Radiation parameter, heat generation parameter, thermophoretic parameter and Schmidt number respectively.

\section{Method OF SOlution}

Equations (15)-(17) constitute highly non-linear coupled boundary value problem of third and secondorder. These systems of non-linear ordinary differential Equations with the relevant boundary conditions Eq. (18) are solved numerically for the velocity, temperature and concentration distributions, by using the Runge-Kutta fourth order along with Shooting method. First of all, higher order non-linear differential Eqs. (15)-(17) are converted into simultaneous linear differential equations of first order and they are further transformed into initial value problem by applying the shooting technique (Jain et al.; 1985). The resultant initial value problem is solved by employing RungeKutta fourth order technique. The step size $\Delta \eta=0.01$ is used to obtain the numerical solution with five decimal place accuracy as the criterion of convergence.

\section{RESULTS AND DISCUSSION}

In order to get a physical insight into the problem, a parametric study is carried out. Throughout the numerical calculations the Prandtl number Pr is chosen as 0.71 which corresponds air at $293 \mathrm{~K}$ and 1 atmosphere of pressure. For numerical computations, the default values of the other parameters considered are $\gamma=2.0, M=0.5, \beta=0.5, R=1.0, E c=0.01$,

$Q=0.1, \tau=1.0, S c=0.6, f_{w}= \pm 0.5$ unless otherwise specified.
In order to assess the accuracy of our computed results, the present result has been compared with Alam et al. (2009) for different values of $M$ on the velocity field is shown Fig. 1 with $\beta=Q=0.0$. It is observed that the agreements with the solution of velocity profiles are excellent.

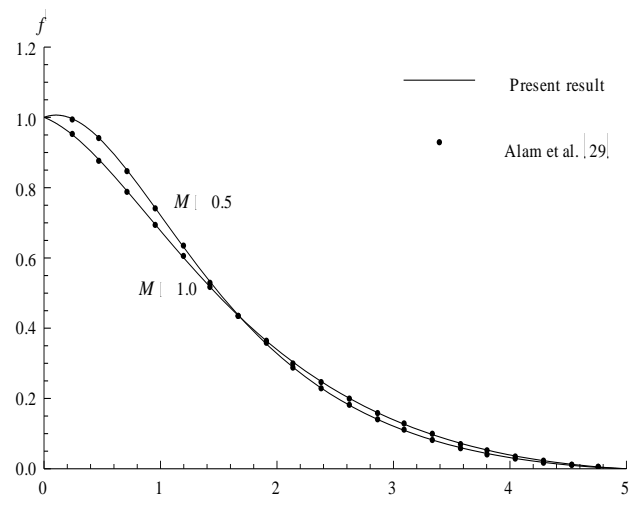

Fig. 1. Comparison of temperature profiles

Figures 2-4, respectively, show the dimensionless velocity, temperature and concentration profiles for different values of magnetic field parameter $M$ for both suction $\left(f_{w}>0\right)$ as well as injection $\left(f_{w}<0\right)$ cases. From Fig. 2 we observe that increasing the magnetic field parameter decreases the velocity inside the boundary layer as a result of the increased retarding force. On the other hand, from Fig. 3 we see that the magnetic field increases the temperature of the fluid inside the boundary layer because of excess heating and consequently decreases the heat flux. Increasing the magnetic field parameter is found to decrease the velocity boundary layer thickness and increase the thermal boundary layer thickness in the case of fluid withdrawing as well as for fluid injecting. Therefore, magnetic field can be used to control the flow and heat transfer characteristics. From Fig. 4 we see that the concentration profile decreases with the increase of the magnetic field parameter.

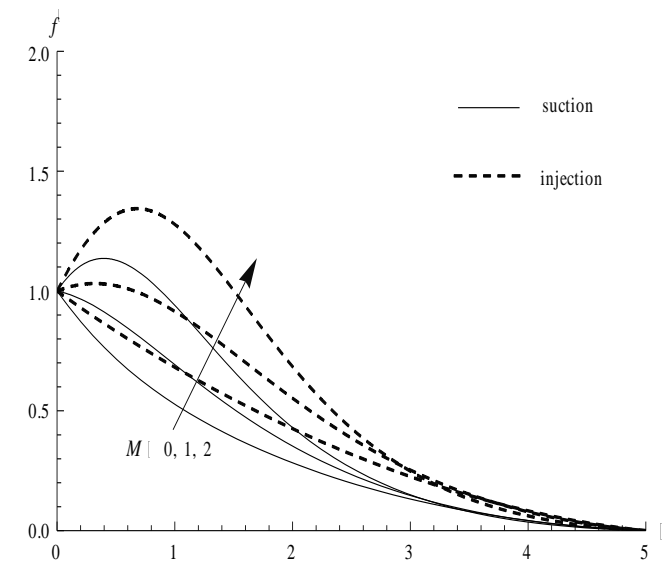

Fig. 2. Velocity profiles for different values of $M$ 


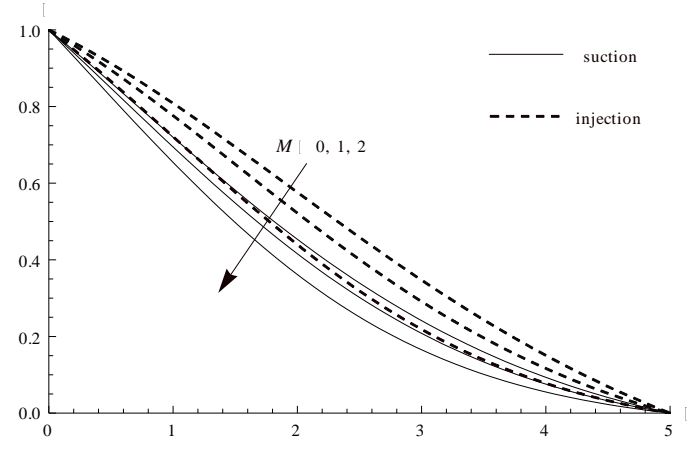

Fig. 3. Temperature profiles for different values of $M$

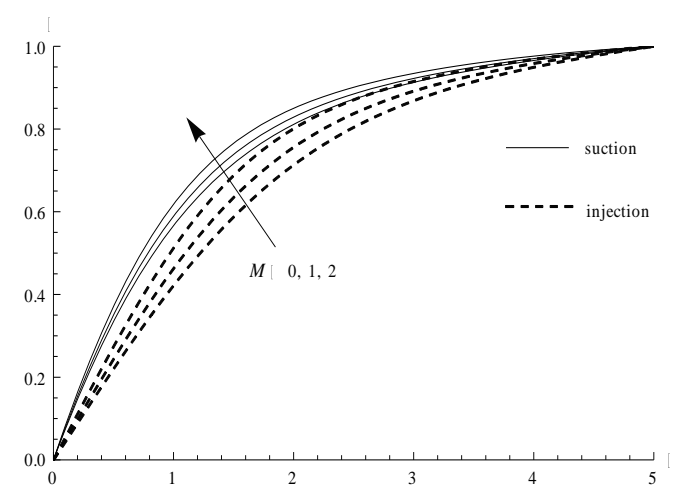

Fig. 4. Concentration profiles for different values of $M$

The effect of buoyancy parameter $\gamma$ on the velocity, temperature and concentration profiles are shown in Figs. 5-7, respectively. It is obvious that an increase in the buoyancy parameter results in increasing velocity and concentration within the boundary layer. As buoyancy parameter increases the temperature decreases.

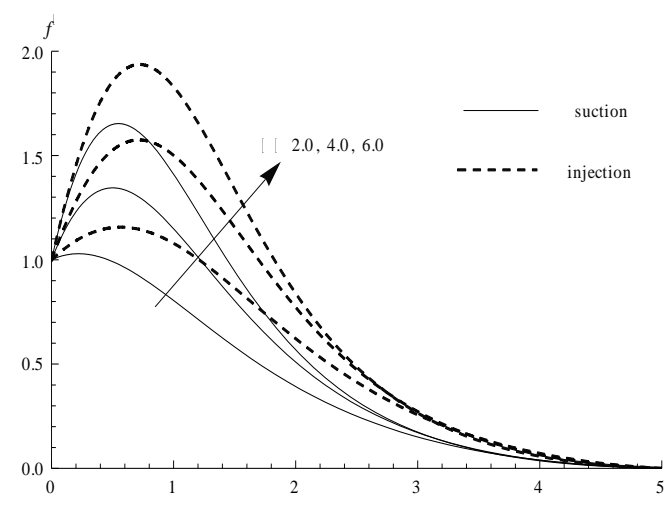

Fig. 5. Velocity profiles for different values of $\gamma$

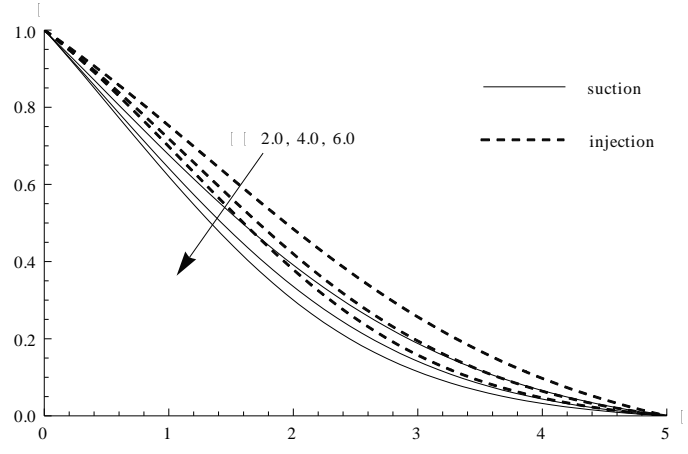

Fig. 6. Temperature profiles for different values of $\gamma$

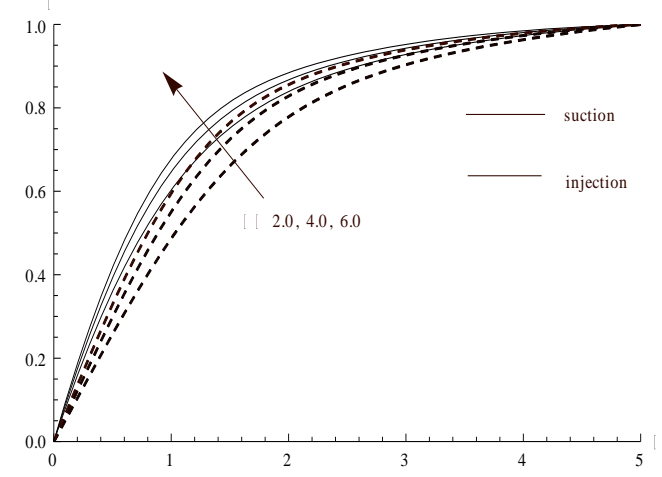

Fig. 7. Concentration profiles for different values of $\gamma$

Figures 8, 9 and 10 show the effects of thermal conductivity parameter $\beta$ on dimensionless velocity, temperature and concentration functions versus transverse coordinate $\eta$. Figure 6 shows that the dimensionless velocity $u$ increase as the thermal conductivity parameter $\beta$ increases. This is because as $\beta$ increases the thermal conductivity of the fluid increases. A rise in $\beta$ from zero (constant thermal conductivity of fluid) through 0.5 to1, induces a significant increase in the temperature in the flow domain (Fig. 9). All profiles decay smoothly to zero from maximum values at the wall to zero in the free stream (edge of the boundary layer). Fluid temperature is therefore maximized with larger values of $\beta$. A similar but less marked trend is seen for the distribution of concentration function with $\eta$ (Fig. 10), where again $\phi$ values are seen to rise with an increase in $\beta$, in particular nearer to the wall. All profiles ascend from zero at the wall to unity in the free stream. 


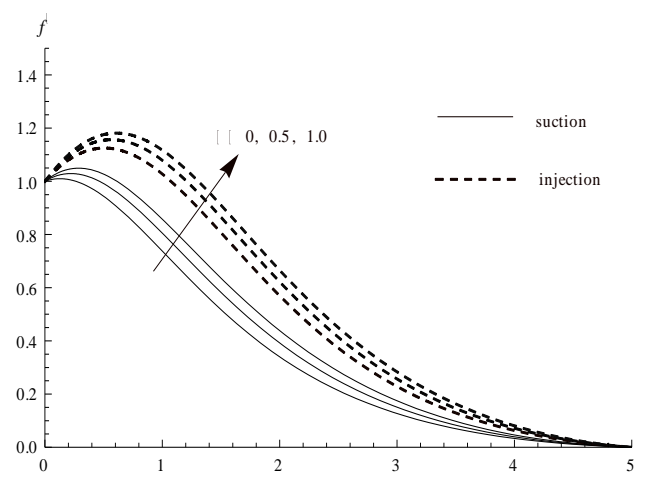

Fig. 8. Velocity profiles for different values of $\beta$

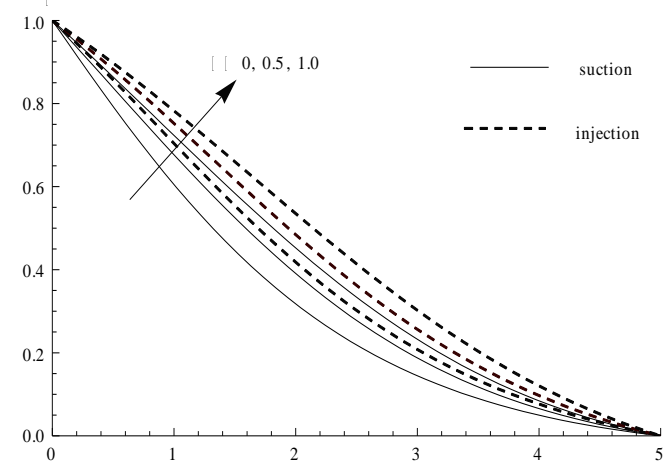

Fig. 9. Temperature profiles for different values of $\beta$

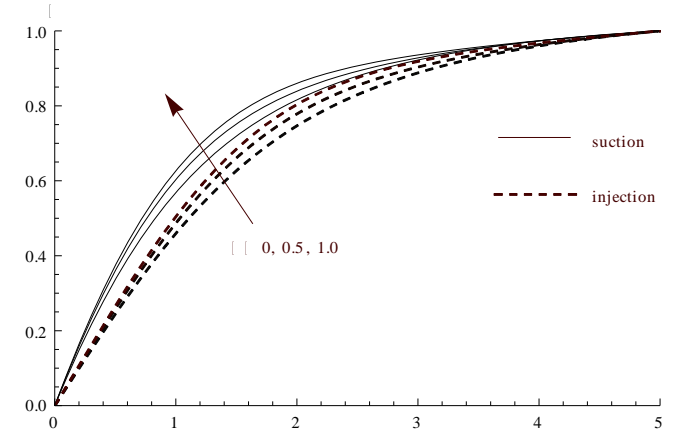

Fig. 10. Concentration profiles for different values of $\beta$

Figures 11-13, respectively, show the dimensionless velocity, temperature and concentration profiles for different values of conduction-radiation parameter $R$. From Fig. 11 we see that increasing the values of $R$ decreases the velocity of the fluid inside the hydrodynamic boundary layer for both case of constant fluid suction or injection. From Fig. 12 it is found that the increasing of conduction-radiation parameter increases the temperature gradients near the porous wall for both case of constant fluid suction or injection, which increases heat transfer rates, this is due to the fact that radiation effect increases temperatures of the fluids and the absence of radiation defines small temperatures. Therefore, radiation intensifies the buoyancy force. Fig. 12 also reveals that for sufficiently strong radiation effect $R \geq 3$ (not precisely determined) in connection with the effect of injection overshoot the temperature profile near the surface of the plate. Fig. 13 shows that decreasing effect of radiation on the concentration profile for fluid suction is less compared to that of fluid injection.

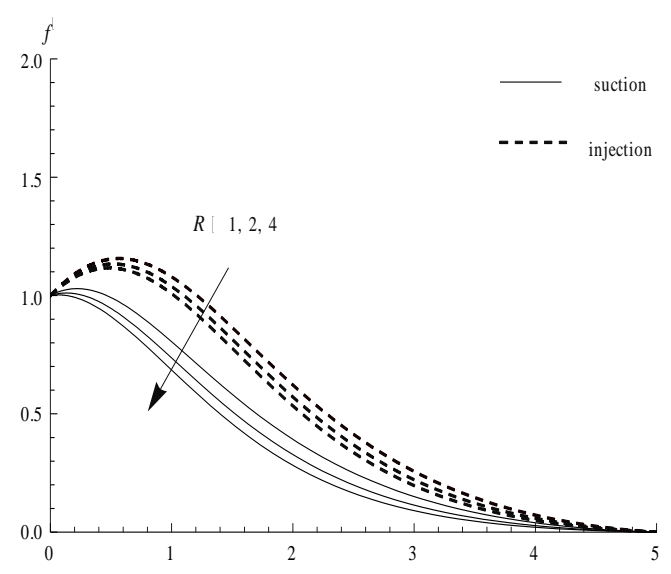

Fig. 11. Velocity profiles for different values of $R$

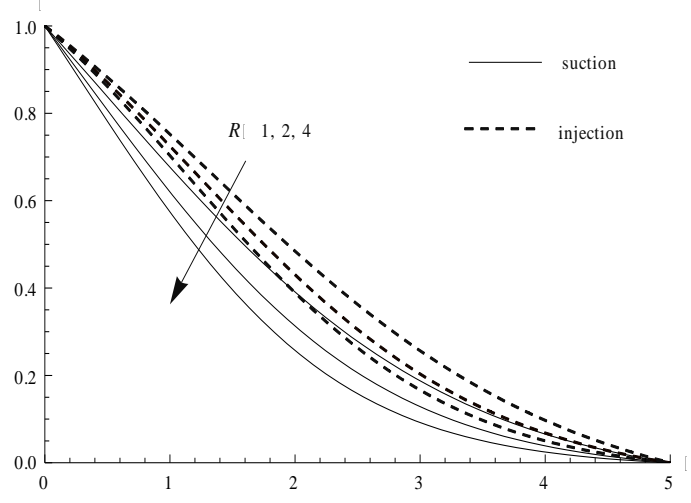

Fig. 12. Temperature profiles for different values of $R$

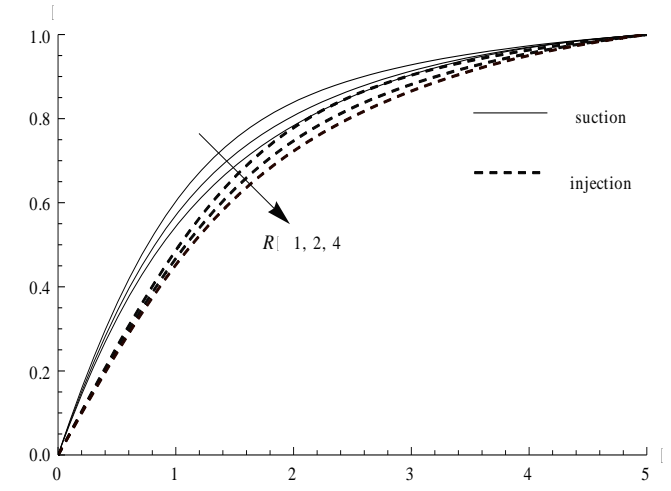

Fig. 13. Concentration profiles for different values of $R$ 
The effect of viscous dissipation parameter or Eckert number $E c$ on the velocity, temperature and concentration profiles are shown in Figs. $14-16$, respectively. These figures reveal that increasing the Eckert number broadens the velocity, temperature as well as concentration distributions inside the velocity, thermal and concentration boundary layers. Increasing effect of $E c$ on the concentration profiles for the case of suction is less pronounceable. But for the case of injection this effect is quite significant.

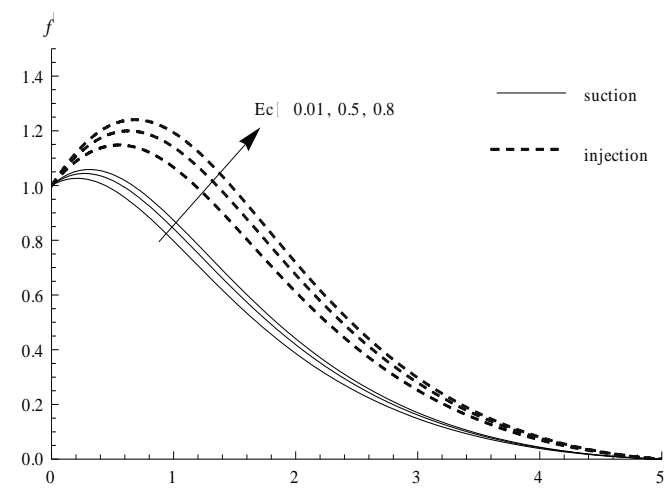

Fig. 14. Velocity profiles for different values of $E c$

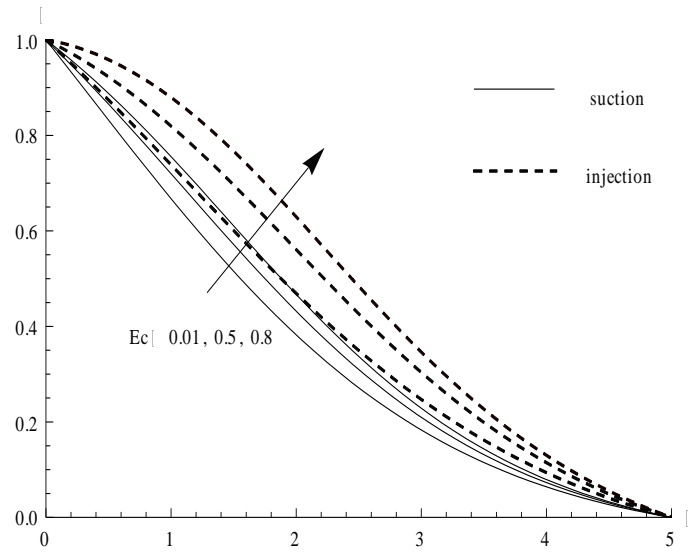

Fig. 15. Temperature profiles for different values of Ec

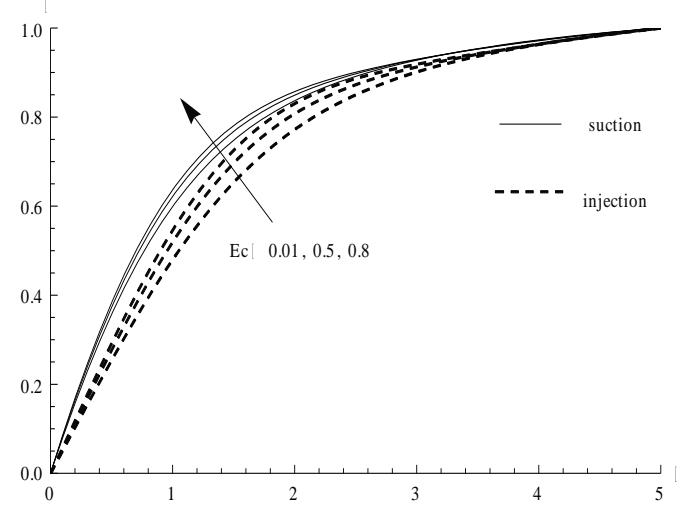

Fig. 16. Concentration profiles for different values of $E c$
Figures 17, 18 and 19 to illustrate the influence of heat generation parameter $Q$ on the dimensionless velocity temperature and concentration fields through the boundary-layer. It is seen from Fig. 17 that the velocity profile is influenced considerably and increases when the value of heat generation parameter increases. As expected a rise in positive value of $Q$ from 0 to 0.5 and 1 induces a clear increase in temperature function $\theta$ throughout the flow domain normal to the plate. Physically heat generation in the fluid will add thermal energy to the flow and therefore for positive $Q$ temperatures will rise. Such a heat source phenomenon is possible in energy system devices or hot spots in industrial treatment systems. In Fig.18, the case of no heat source logically lies at the interface between the minimal values of positive $Q$. The trend for all temperature plots, in consistency with the wall and free stream boundary conditions is a gradual decay from the plate (wall) to the edge of the boundary layer, where we observe all profiles converging to zero at approximately $\eta=5$. These effects are very similar to nonmagnetic studies, indicating that heat source effects are not influenced by the presence of a transverse magnetic field. A similar but less dramatic trend is observed for the distribution of concentration $\phi$ in the domain (Fig. 19). Again $\phi$ is increased with a positive rise in $Q$ i.e. increasing heat generation.

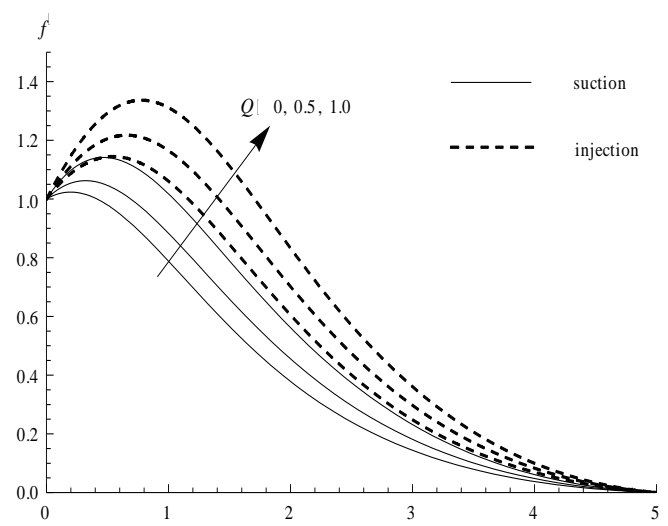

Fig. 17. Velocity profiles for different values of $Q$

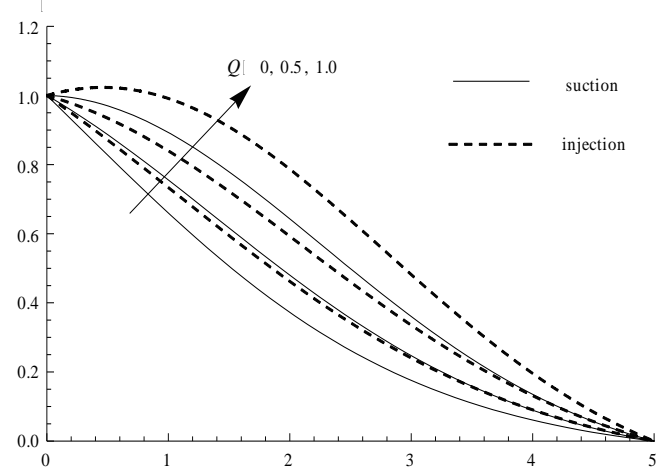

Fig. 18. Temperature profiles for different values of $Q$ 


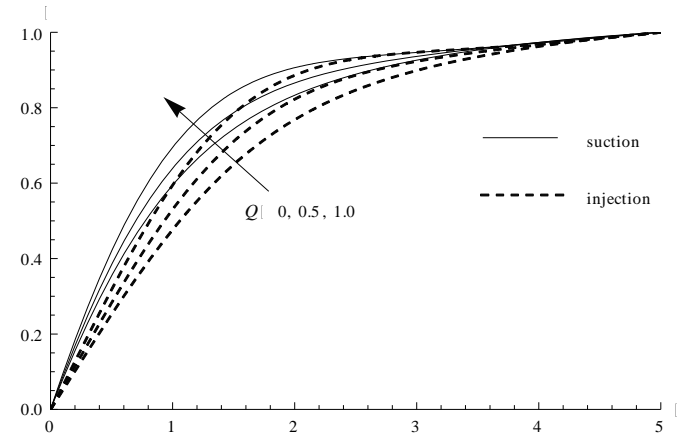

Fig. 19. Concentration profiles for different values of $Q$

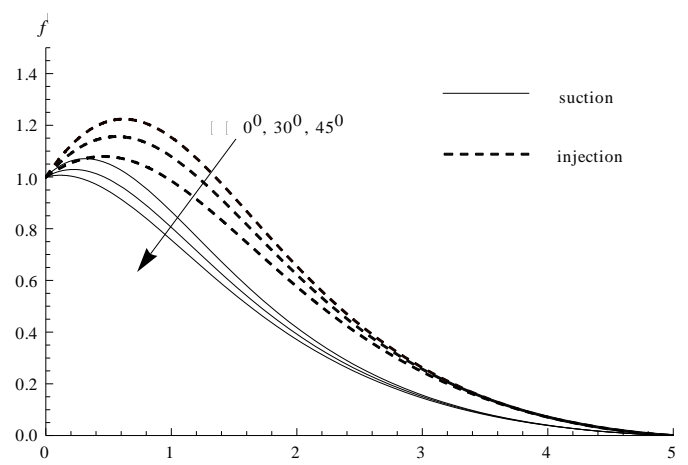

Fig. 20. Velocity profiles for different values of $\alpha$

Representative velocity profiles for three typical angles of inclination ( $\alpha=0^{0}, 30^{\circ}$ and $45^{\circ}$ ) are presented in Fig. 20 for both fluid suction as well as injection cases. It is observed from Fig. 20 that increasing the angle of inclination decreases the velocity inside the hydrodynamic boundary layer. This is because the angle of inclination decreases the effect of the buoyancy force due to thermal diffusion by a factor of $\cos \alpha$. Consequently the driving force to the fluid decreases as a result velocity of the fluid decreases. The velocity of the injecting fluid amplifies the fluid velocity within the boundary layer. The combined effects of injection and the buoyancy force (which is maximum for $\alpha=0$ ) overshoots the main stream velocity significantly. From Figs. 21-22 we observe that both the thermal as well as concentration boundary layer thickness increases as the angle of inclination increases. The increasing effect of a on these boundary layers thickness is quite strong for the case of injection. The effect of thermophoretic parameter $\tau$ on the concentration field is shown in Fig. 23. From this figure we observe that concentration boundary layer thickness increases as the thermophoretic parameter increases for fluid suction as well as for fluid injection.

Figure 24 shows concentration profiles for various values of the Schmidt number $S c$. From this figure it is clear that the concentration boundary layer thickness decreases as the Schmidt number $S c$ increases that analogous to the effect of increasing the Prandtl number on the thickness of a thermal boundary layer.

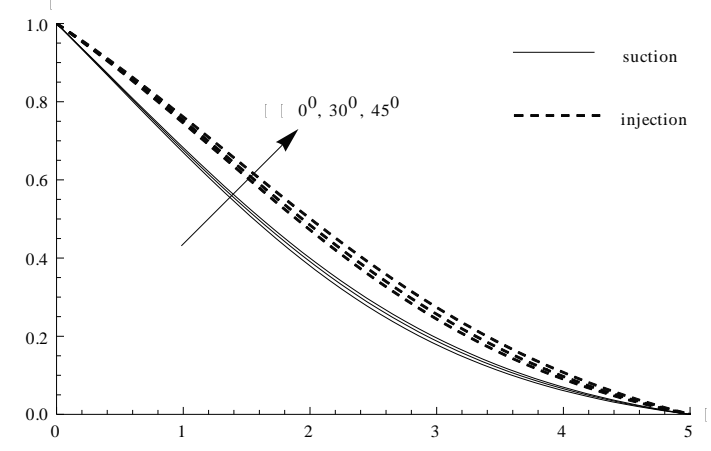

Fig. 21. Temperature profiles for different values of $\alpha$

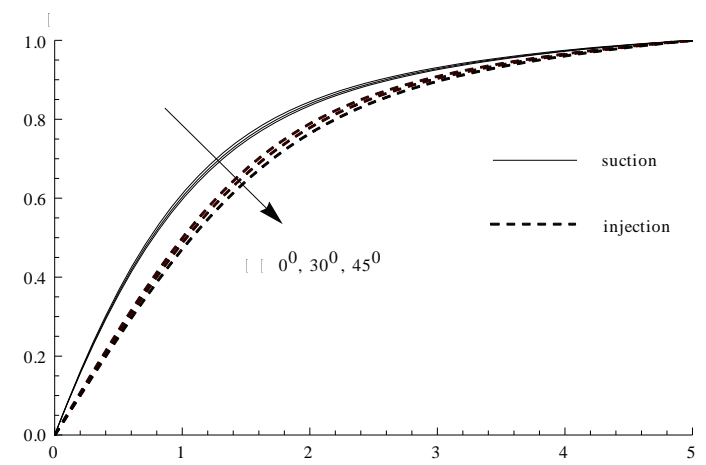

Fig. 22. Concentration profiles for different values of $\alpha$

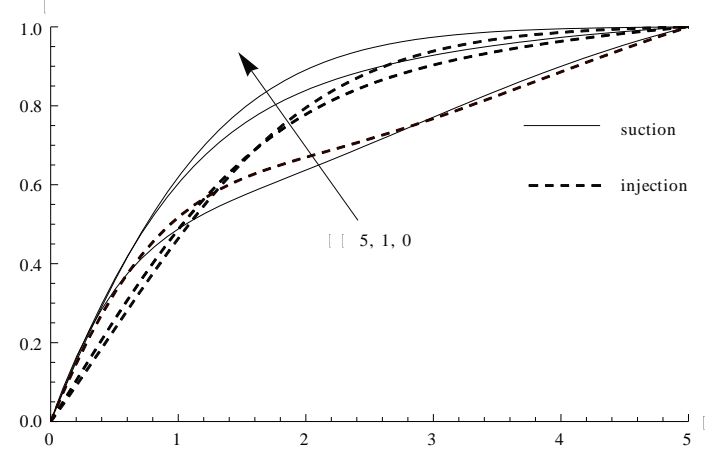

Fig. 23. Concentration profiles for different values of $\gamma$

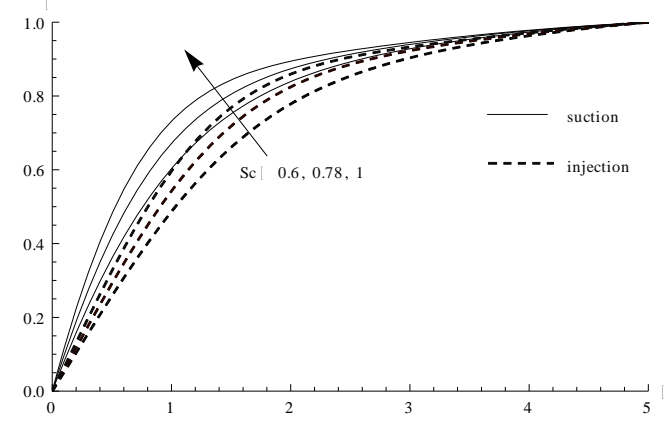

Fig. 24. Concentration profiles for different values of Sc 


\section{Conclusion}

In this article a mathematical model has been presented for the hydromagnetic boundary layer flow over an inclined radiate isothermal permeable surface with thermophoresis present and also heat generation effects and viscous and Joule heating with variable. Using transformations a set of ordinary differential equations has been derived for the conservation of mass, momentum and species diffusion in the boundary layer regime. These nonlinear, coupled differential equations have been solved under physically valid boundary conditions using the Runge-Kutta fourth order along with shooting method. . Fluid velocity and fluid temperature increase with the increase in parameter $\beta$.Magnetic field has been shown in the present flow scenario to infact induce acceleration of the flow, rather than deceleration, but to reduce temperatures and increase concentration of particles in the boundary layer. Fluid velocity decreases with the application of suction and increases in case of injection of fluid in the boundary layer. A positive increase in Eckert number is shown to reduce temperatures in the flow, as experienced via both viscous dissipation and Joule (Ohmic) heating. Themophoresis for the case of a cold wall (positive $\tau$ ) is shown to initially increase concentration of particles in the boundary layer, but a short distance from the wall this trend is reversed. The computations have important implications in aerosol deposition dynamics, hydronautics of blades, and also optical fiber manufacture under magnetic field control.

\section{REFERENCES}

Alam, M.S., M.M. Rahman and M.A. Sattar (2009). On the effectiveness of viscous dissipation and Joule heating on steady Magnetohydrodynamic heat and mass transfer flow over an inclined radiate isothermal permeable surface in the presence of thermophoresis. Comm. Nonlinear Sci. Numerical Simulation, 14, 2132-2143.

Alam, M.S., M.M. Rahman and M.A. Sattar (2008). Effects of variable suction and thermophoresis on steady MHD combined free-forced convective heat and mass transfer flow over a semi-infinite permeable inclined plate in the presence of thermal radiation, Int. J. Thermal Sci., 47, 758-765.

Batchelor, G.K. and C. Shen (1985). Thermophoretic deposition of particles in gas flowing over cold surfaces. J. Colloid. Interf. Sci., 107(1), 21-37.

Brock, J.R. (1962). On the theory of thermal forces acting on aerosol particles. J. Colloid. Sci., 17, 768-780.

Chamkha, A.J. and I. Camille, (2000). Effects of heat generation/absorption and thermophoresis on hydromagnetic flow with heat and mass transfer over a flat surface. Int. J. Numerical Methods in Heat and Fluid Flow, 10, 432-448.
Duwairi, H.M. (2005). Viscous and joule heating effects on forced convection flow from radiate isothermal porous surfaces, Int. J. Numerical Methods in Heat Fluid Flow, 15, 429-440.

Duwairi, H.M., R.A. Damseh, (2004). Magnetohydrodynamic natural convection heat transfer from radiate vertical porous surfaces. Heat Mass Trans, 40, 787-792.

Duwairi, H.M. and R.A. Damseh (2004). MHDbuoyancy aiding and opposing flows with viscous dissipation effects from radiate vertical surfaces. Can. J. Chem Eng, 82, 613-618.

Gnaneswara Reddy, M. (2013). Lie group analysis of heat and mass transfer effects on steady MHD free convection flow past an inclined surface with viscous dissipation. Journal of Applied Fluid Mechanics, 6(3), 397-404.

Gnaneswara Reddy, M. (2012). Lie group analysis of heat and mass transfer effects on steady MHD free convection dissipative fluid flow past an inclined porous surface with heat generation. Theoret. Appl. Mech., 39(3), 233-254.

Gnaneswara Reddy, M. and N. Bhaskar Reddy (2011). Mass transfer and heat generation effects on MHD free convection flow past an inclined vertical surface in a porous medium. Journal of Applied Fluid Mechanics, 4 (3), 7-11.

Gnaneswara Reddy, M. and N. Bhaskar Reddy (2010). Radiation and mass transfer effects on unsteady MHD free convection flow past a vertical porous plate with viscous dissipation. Int. J. Appl. Math and Mech., 6(6), 96-110.

Goren, S.L. (1977). The role of thermophoresis of laminar flow of a viscous and incompressible fluid. J. Colloid. Interf. Sci., 61, 77.

Hossain, M.A., M.A. Alim and D.A.S. Rees (1999). The effect of radiation on free convection from a porous vertical plate. Int J Heat Mass Trans, 42, 181-191.

Hossain, M.A., H.S. Takhar (1996). Radiation effects on mixed convection along a vertical plate with uniform surface temperature. Heat Mass Trans, 31, 243-248.

Jain, M.K., S.R.K. Iyengar and R.K. Jain (1985). Numerical Methods for Scientific and Engineering Computation. Wiley Eastern Ltd., New Delhi.

Kanki, T., S. Iuchi, T. Miyazaki and H. Udea (1985). On thermophoresis of relatively large aerosol particles suspended near a plate. J. Colloid. Interf. Sci., 107(2), 418-425.

Kremer, D. M., R.W. Davis, E.F. Moore, J.E. Maslar, D.R. Burgessmand S.H. Ehrman (2003). J. Electrochemical Society, 150(2), G127-G139. 
Lin, B.Y.H. and K. Ahn (1987). Particle deposition on semiconductor wafers. Aerosol Sci. Tech., 6, 215224.

Mbeledogu, I.U. and A. Ogulu (2007). Heat and mass transfer of an unsteady MHD natural convection flow of a rotating fluid past a vertical porous flat plate in thepresence of radiative, heat transfer. Int. J. Heat Mass Trans , 50, 1902-1908.

Mills, A.F., X. Hang and F. Ayazi (1984). The effect of wall suction and thermophoresis on aerosolparticle deposition from a laminar boundary layer on a flat plate. Int. J. Heat Mass Transfer, 27, $1110-1114$.

Osalusi, E., J. Side, R. Harris and B. Johnston (2007). On the effectiveness of viscous dissipation and Joule heating on steady MHD flow and heat transfer of a Bingham fluid over a porous rotating disk in the presence of Hall and ion-slip currents, Int. Comm. Heat Mass Trans, 34, 1030-1040.
Rahman, M.M. and M.A. Sattar (2007). Transient convective flow of micropolar fluid past a continuously moving vertical porous plate in the presence of radiation. Int. J. Appl Mech Eng, 12, 497-513.

Sasse, A.G.B.M., W.W. Nazaroff and A.J. Gadgil (1992, October). Thermophoretic removal of particles from laminar flow between parallel plates and concentric tubes. Am. Assoc. Aerosol. Res. Conf., San Francisco, California.

Shen, C. (1998). Thermophoretic deposition of particles onto cold surface of bodies in two dimensional and axi-symmetric flows. J. Colloid. Interf. Sci., 127, 104-115.

Talbot, L., R.K. Cheng, R.W. Schefer and D.R. Willis (1980). Thermophoresis of particles in a heated boundary layer. J. Fluid Mech., 101(4), 737-758.

Tsai, R. (1999), A simple approach for evaluating the effect of wall suction and thermophoresis on aerosol particle deposition from a laminar flow over a flat plate. Int. Comm. Heat Mass Transfer, 26, 249-257. 